\title{
Comparison of Efficacy of LUS and CXR in the Diagnosis of Children Presenting with Respiratory Distress to Emergency Department
}

\author{
Laila M Hegazy ${ }^{1}$, Ahmed R Rezk², Hossam M Sakr³, Asmaa S Ahmed ${ }^{4}$
}

\begin{abstract}
Introduction: Respiratory distress (RD) in children is a life-threatening condition. Delay in diagnosis has a deleterious effect on morbidity and mortality. The bedside lung ultrasound in emergency (BLUE) is a fast method that aims to accelerate the diagnosis with minimal radiological exposure. We targeted to evaluate the efficacy of BLUE protocol to speed and increase the precision of recognizing the cause of RD compared with chest X-ray (CXR) in the emergency department.

Materials and methods: A cross-sectional study on 63 children with RD attended the emergency of a tertiary, university-affiliated, pediatric medical center between January 2017 and January 2018.

Results: Most cases were males 52.4\%. We designed to estimate the value of BLUE as a diagnostic tool for RD and comparing it with CXR. Pneumonia with or without pleural effusion was the main etiology of RD detected by BLUE in $47.7 \%$ of studied children, pulmonary edema in $22.2 \%$, bronchiolitis and asthma in $17.4 \%$, and pneumothorax in $12.7 \%$. Lung ultrasound (LUS) was superior to CXR in the diagnosis of RD cause, and most cases, $47.7 \%$ were diagnosed with pneumonia with a sensitivity of $93.5 \%$ and specificity $96.9 \%$.

Conclusion: Bedside lung ultrasound in emergency is an effective tool for identifying the cause of RD which is more sensitive and specific compared with CXR.

Keywords: Bedside lung ultrasound in emergency protocol, Chest X-ray, Emergency, Pediatrics.

Indian Journal of Critical Care Medicine (2020): 10.5005/jp-journals-10071-23459
\end{abstract}

\section{INTRODUCTION}

In intensive care units (ICUs), thoracic imaging is applied to most of the patients. The mobile standard chest X-ray (CXR) is one of the most attainable imaging modalities. However, CXR may be incovenient for critically ill children in the emergency department and carry possible hazards of irradiation. ${ }^{1}$ The CXR procedures take a significant amount of time which may not be available. The good quality of the CXR image is not always warranted. Chest $\mathrm{X}$-ray interpretation can be challenging if critically ill patients have multiple radiographic abnormalities. ${ }^{2}$ Technical barriers may lead to inaccurate assessment of lung consolidation, pleural effusion, and alveolar-interstitial syndrome. ${ }^{3}$

Lung ultrasound (LUS) is a safe and efficient tool for emergency diagnosis of respiratory distress (RD). Bedside lung ultrasound in emergency (BLUE) protocol can be used as a screening tool in acutely dyspneic or hypoxic children to provide an instant diagnosis and influence therapeutic intervention. ${ }^{1}$ This reduces the need for more hazardous tools as CXR and computerized tomography (CT) scans. ${ }^{2}$ The protocol includes the study of lung areas to detect ultrasound findings resulting in seven profiles that are specific to the corresponding clinical diagnosis. This protocol is a part of an approach that includes basic history, physical examination, and basic laboratory tests.

In our study, we examined children aging between 1 month and 18 years admitted to the emergency department with RD. Our study aims to evaluate the accuracy of BLUE protocol in identifying the cause of RD in comparison with CXR through seven different lung profiles in the Emergency Department of Ain Shams Hospitals. 1,2,4 Department of Pediatrics, Ain Shams University Hospital, Cairo,
Egypt
${ }^{3}$ Department of Radiology, Ain Shams University Hospital, Cairo,
Egypt

Corresponding Author: Ahmed R Rezk, Department of Pediatrics, Ain Shams University Hospital, Cairo, Egypt, Phone: +20 1113311183, e-mail: ahmed_rezk@med.asu.edu.eg

How to cite this article: Hegazy LM, Rezk AR, Sakr HM, Ahmed AS. Comparison of Efficacy of LUS and CXR in the Diagnosis of Children Presenting with Respiratory Distress to Emergency Department. Indian J Crit Care Med 2020;24(6):459-464.

Source of support: Nil

Conflict of interest: None

\section{Materials and Methods}

A cross-sectional observational study on 63 children with RD admitted to the emergency room, Children's Hospital, Ain Shams University. The study started in January 2017 and was fulfilled in January 2018. Informed consent was signed by the parents or guardians of all the participants for admission to the hospital and the procedures performed during the hospitalization.

Inclusion criteria were children $>1$ month and $<18$ years with RD defined as dyspnea, tachypnea $>60$ breaths/minute or tachypnea for age, severe hypoxemia in room air and/or cyanosis, pulse oximetry $\mathrm{SpO}_{2}<92 \%$, grunting, apnea, working accessory muscles as intercostals, and nose flaring.

(c) The Author(s). 2020 Open Access This article is distributed under the terms of the Creative Commons Attribution 4.0 International License (https://creativecommons. org/licenses/by-nc/4.0/), which permits unrestricted use, distribution, and non-commercial reproduction in any medium, provided you give appropriate credit to the original author(s) and the source, provide a link to the Creative Commons license, and indicate if changes were made. The Creative Commons Public Domain Dedication waiver (http://creativecommons.org/publicdomain/zero/1.0/) applies to the data made available in this article, unless otherwise stated. 
Exclusion criteria were age $\leq 1$ month or $\geq 18$ years, and/or patients having a prior diagnosis to avoid bias. Also, patients and/ or relatives not giving consent were excluded. The initial screening for children with RD was made by the emergency team using standardized clinical tests before comparing and confirming the results of BLUE protocol and CXR. The main diagnoses included pneumonia with or without pleural effusion, acute hemodynamic lung edema, obstructive lung disease (bronchiolitis or asthma), and pneumothorax.

Included patients were subjected to full history and clinical examination. Recorded vital signs were temperature, respiratory rate, heart rate, blood pressure, complexion, consciousness level, capillary refill time, and chest examination. Routine laboratory tests were done for all the patients including complete blood count (CBC) (by Sysmex XE-2100, Kobe, Japan) and C-reactive protein (CRP) by the latex agglutination test (Omega, UK). Radiological evaluations were CXR anteroposterior (AP) view as the patients were in a supine position. The LUS using BLUE protocol was done within 1 hour of admission after the initial screening. The BLUE protocol was established to know the cause of RD. ${ }^{4}$ The LUS was performed by the same experienced radiologist using (SonoScape S8) with a linear lens and virtual convex ability which can visualize deeper lung structures. The radiologist, who did and reported both the CXR and LUS, was blinded to the clinical and laboratory diagnoses of the patients. The evaluation was in a semi-recumbent position or supine position. The LUS results were interpreted according to the original BLUE protocol study. The LUS images were obtained for each of the six quadrants in each hemithorax (upper and lower parts of the anterior, lateral, posterior chest wall, delimited by anterior and posterior axillary lines). All quadrants were categorized according to the predominant profiles $(A, B$, or $C$ ) as shown in Table 1. Lung sliding is an essential ultrasound finding to identify normal lung aeration, and it indicates the regular movement of the pleural line (described as a bright white line) in regular cycles in synchrony with each respiratory movement. Following the original BLUE protocol in order not to miss the diagnosis of pulmonary embolism, normal profile (bilateral lung sliding with A-lines) should be combined

Table 1: Lung ultrasound profiles

\begin{tabular}{lll}
\hline Profile & Description & Indicates \\
\hline A (A-lines) & $\begin{array}{l}\text { Static horizontal white lines ap- } \\
\text { pear at regular intervals } \\
\text { Absence of lung sliding combined } \\
\text { with the presence of A-lines }\end{array}$ & $\begin{array}{l}\text { Asthma or } \\
\text { bronchiolitis } \\
\text { Pneumothorax }\end{array}$ \\
A & $\begin{array}{l}\text { In addition to venous thrombosis } \\
\text { B }\end{array}$ & $\begin{array}{l}\text { Pulmonary } \\
\text { embolism }\end{array}$ \\
& $\begin{array}{l}\text { Hyperechoic vertical artifacts } \\
\text { that move in synchrony with the } \\
\text { respiratory cycle }\end{array}$ & Pulmonary edema \\
B' & $\begin{array}{l}\text { Absence of lung sliding combined } \\
\text { with diffuse anterior lung rockets }\end{array}$ & Pneumonia \\
A/B & $\begin{array}{l}\text { A half A profile in one lung and a } \\
\text { half B profile in the other }\end{array}$ & Pneumonia \\
& $\begin{array}{l}\text { A tissue-like structure contains } \\
\text { white points or air bronchogram } \\
\text { C }\end{array}$ & $\begin{array}{l}\text { Anterior lung } \\
\text { consolidation }\end{array}$ \\
& $\begin{array}{l}\text { Pneumonia with or without } \\
\text { pleural syndrome }\end{array}$ & \\
PLAPS &
\end{tabular}

with screening for leg vein thrombosis (which was not performed in our study). ${ }^{4,5}$

\section{Statistical Methods}

The sample size was calculated using PASS 11.0 sample size calculation program. It was calculated based on the least sensitivity and specificity for ultrasound in critically ill dyspneic patients. The sample included 63 patients to achieve $90 \%$ power to detect a change in sensitivity from 0.5 to 0.81 using a two-sided binomial test and to detect a change in specificity from 0.5 to 1.00 using a two-sided binomial test. The significance level achieved by the sensitivity test is 0.0414 and achieved by the specificity test is 0.5000 . The prevalence of the disease is 0.97 .

These calculations were based on the findings retrieved from a study carried out by Lichtenstein and Meziere. ${ }^{4,6}$ They found out that predominant A-lines plus lung sliding indicated asthma and had $89 \%$ sensitivity and $97 \%$ specificity. Multiple anterior diffuse B-lines with lung sliding, which indicated pulmonary edema, had $97 \%$ sensitivity and $95 \%$ specificity. Anterior absent lung sliding plus A-lines plus lung point indicated pneumothorax with $81 \%$ sensitivity and $100 \%$ specificity. Anterior alveolar consolidations, anterior diffuse B-lines with abolished lung sliding, anterior asymmetric interstitial patterns, posterior consolidations, or effusions without anterior diffuse B-lines indicated pneumonia with $89 \%$ sensitivity and $94 \%$ specificity.

\section{Statistical Analysis}

Data were tabulated and analyzed using Statistical Package for Social Sciences (SPSS) (IBM 20.0). Data were presented, and a suitable analysis was done according to the type of data obtained for each parameter: Mean, standard deviation, and range for parametric numerical data, while median and interquartile range (IQR) for nonparametric data, and frequency and percentage for non-numerical data.

Diagnostic accuracy of ultrasound as compared to clinical diagnosis will be determined with sensitivity, specificity, positive predictive value (PPV), and negative predictive value (NPV).

\section{Results}

The study comprised 63 patients who met the inclusion criteria, 30 (47.6\%) males and 33 (52.4\%) females. Table 2 shows the baseline characteristics of the study participants. According to the bedside lung ultrasound with clinical diagnosis, community-acquired pneumonia with or without pleural syndrome $47.7 \%$ was the main etiology for RD in children [B', C, A/B, posterolateral alveolar and/ or pleural syndrome (PLAPS) profiles], while pulmonary edema or interstitial syndrome was the second most common etiology (B profile) in $22.2 \%$ of studied children. Bronchiolitis and asthma (A profile) were diagnosed in $17.4 \%$ of patients with RD, while pneumothorax $12.7 \%$ ( $A^{\prime}$ profile with lung point) was the least common cause (Table 3 and Figs 1 to 4 ). Table 4 shows the diagnosis according to the $\mathrm{X}$-ray in relation to clinical diagnosis, while Table 5 shows the diagnosis depending on clinical examination only.

\section{Discussion}

The information provided by LUS can add to the physical assessment and clinical expectations. The main advantages were being safe, quick, and bedside too. ${ }^{7}$ Many studies discussed the use of LUS in the PICU, NICU, and AICU, but few studies reported 
the benefits of using this tool in the emergency room (ER) as a bedside test. The ultrasound usage for instant management of life-threatening conditions is one of the main improvements over the years. ${ }^{5}$ We studied the accuracy of LUS in the diagnosis of the commonly encountered causes of RD in the pediatric emergency. Our results showed a satisfactory outcome of LUS in the patients with RD admitted to the emergency using the BLUE protocol and were superior to the results of the X-ray ${ }^{8}$ (Tables 6 and 7).

In our series, pneumonia was the most common cause of RD in children $47.7 \%$. Of the 31 children with a confirmed diagnosis of pneumonia using BLUE protocol, LUS showed the findings consistent with pneumonia in 30 children including 12 patients with para-pneumonic pleural effusion (10 C profile, $9 \mathrm{~B}^{\prime}$ profile, 8 A profile with PLAPS, 3 A/B profile) with a sensitivity of $93.5 \%$, specificity $96.9 \%$, PPV 96.7\%, negative predictive value (NPV) $93.9 \%$ as measured against the final diagnosis, while CXR was positive for pneumonia in 28 children and only 5 patients with effusion with a sensitivity of $90.3 \%$ and specificity $87.5 \%$, PPV $87.5 \%$, NPV $90.3 \%$.

Table 2: Characteristics of study participants

\begin{tabular}{lll}
\hline Characteristic & Mean & $\begin{array}{l}\text { Standard } \\
\text { deviation }\end{array}$ \\
\hline Age in months, median [IQR] & 12 & {$[4-24]$} \\
Order of birth & 2.9 & \pm 1.44 \\
Hemoglobin, mg/dL & 9.6 & \pm 1.27 \\
Platelets, per microliter & 276,342 & $\pm 192,459$ \\
White blood cells, per microliter & 17,271 & $\pm 10,197$ \\
CRP, mg/L, median [IQR] & 12 & {$[6-31.7]$} \\
BUN, mg/dL, median [IQR] & 9 & {$[6-23.5]$} \\
Creatinine, mg/dL, median [IQR] & 0.4 & {$[0.3-0.6]$} \\
$\mathrm{Na}^{+}, \mathrm{mEq} / \mathrm{L}$ & 135.5 & \pm 6.5 \\
$\mathrm{~K}^{+}, \mathrm{mEq} / \mathrm{L}$ & 4.3 & \pm 1.14 \\
$\mathrm{Ca}{ }^{2+}, \mathrm{mg} / \mathrm{dL}$ & 8.9 & \pm 0.75 \\
$\mathrm{PT}$, second, median [IQR] & 14 & {$[13-15.5]$} \\
$\mathrm{PTT}$, second, median [IQR] & 38 & {$[32.7-45]$} \\
\hline
\end{tabular}
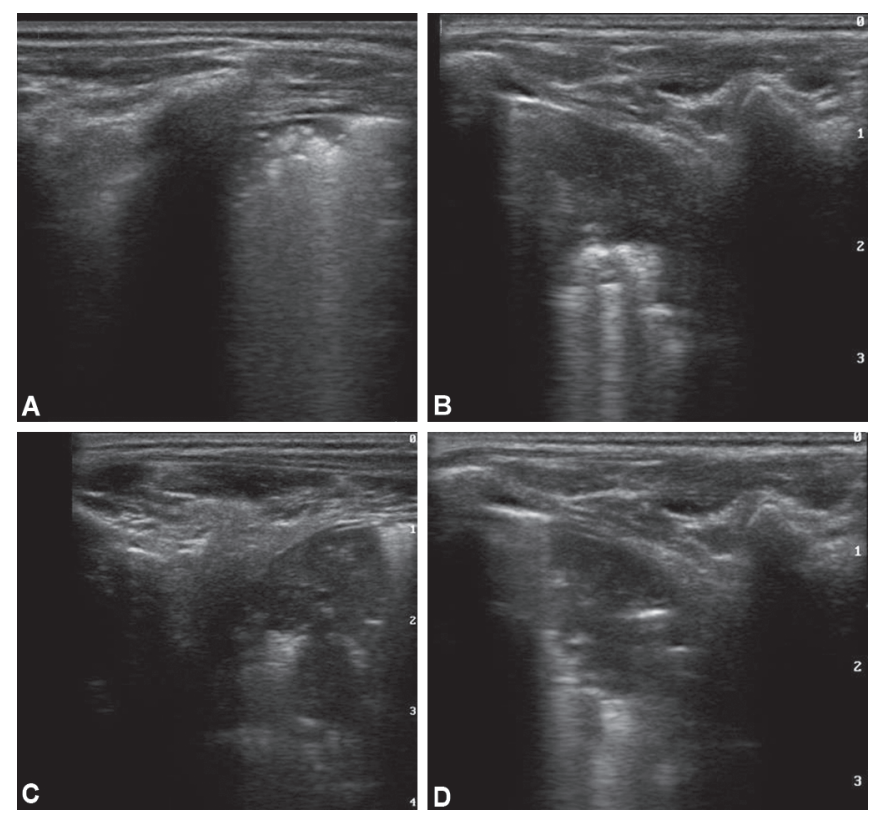

Figs $1 A$ to $D$ : Anterior lung consolidation C profile
Pneumonia was more common in females than in males. In the present study, one patient was given the B' profile by ultrasound so was given the diagnosis of pneumonia but was clinically diagnosed as pulmonary edema, while another patient was given the B profile by ultrasound; hence, the diagnosis of pulmonary edema but clinically diagnosed as pneumonia. Distinguishing pulmonary edema and interstitial pneumonia is yet a limitation of this ultrasound approach, hence the naming interstitial syndrome. Clinical data combined with ultrasound might increase ultrasound sensitivity and specificity.

Table 3: Bedside lung ultrasound in the studied patients in relation to clinical diagnosis

\begin{tabular}{llrl}
\hline Clinical diagnosis & BLUE protocol & No. & Percentage (\%) \\
\hline Pneumonia profiles & C profile & 10 & 15.9 \\
& B' profile & 9 & 14.3 \\
& A with PLAPS & 8 & 12.7 \\
& A/B profile & 3 & 4.8 \\
& & 30 & 47.7 \\
$\begin{array}{l}\text { Pulmonary edema } \\
\text { profile }\end{array}$ & B profile & 14 & 22.2 \\
$\begin{array}{l}\text { Obstructive lung } \\
\text { disease }\end{array}$ & A nor B nor PLAPS & 11 & 17.4 \\
$\begin{array}{l}\text { Asthma or } \\
\text { bronchiolitis }\end{array}$ & profile (nude profile) & & \\
$\begin{array}{l}\text { Pneumothorax } \\
\text { Aneum }\end{array}$ & A with lung point & 8 & 12.7 \\
& profile & & \\
\hline
\end{tabular}
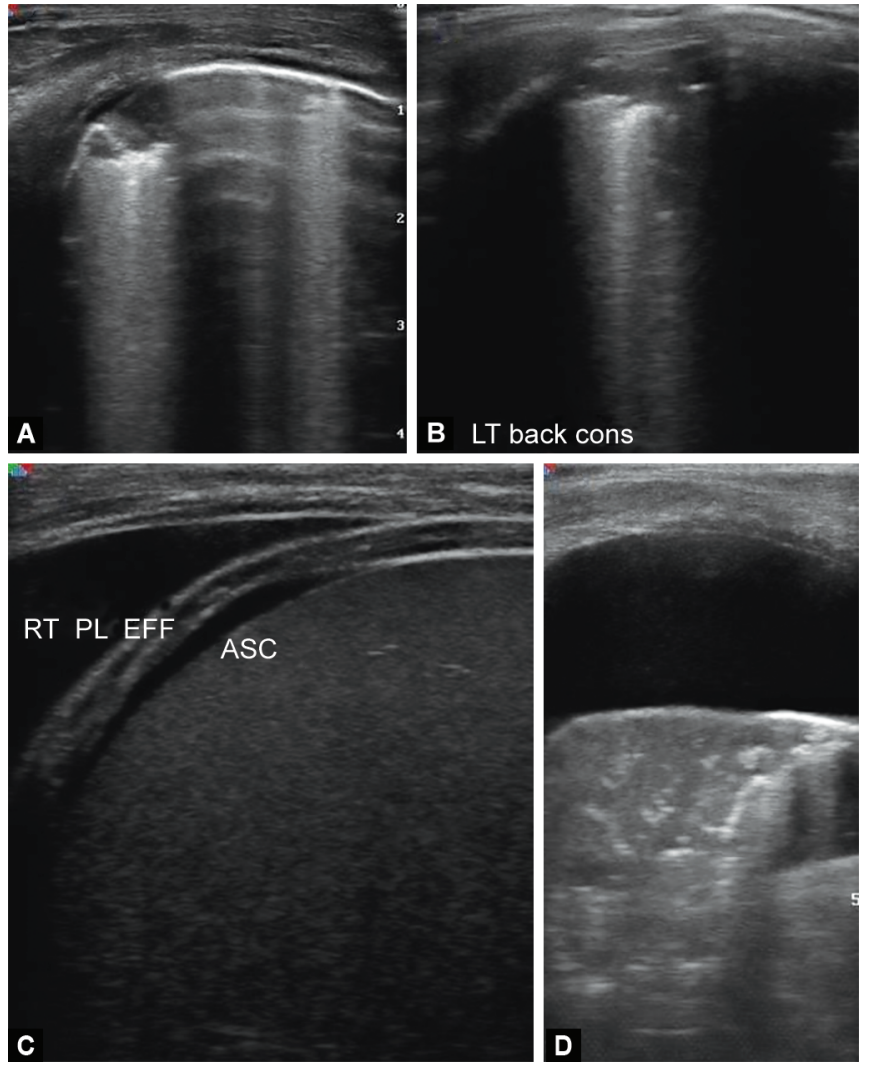

Figs 2A to D: RT pleural effusion (C) associated with lung consolidation (D) PLAPS profile 

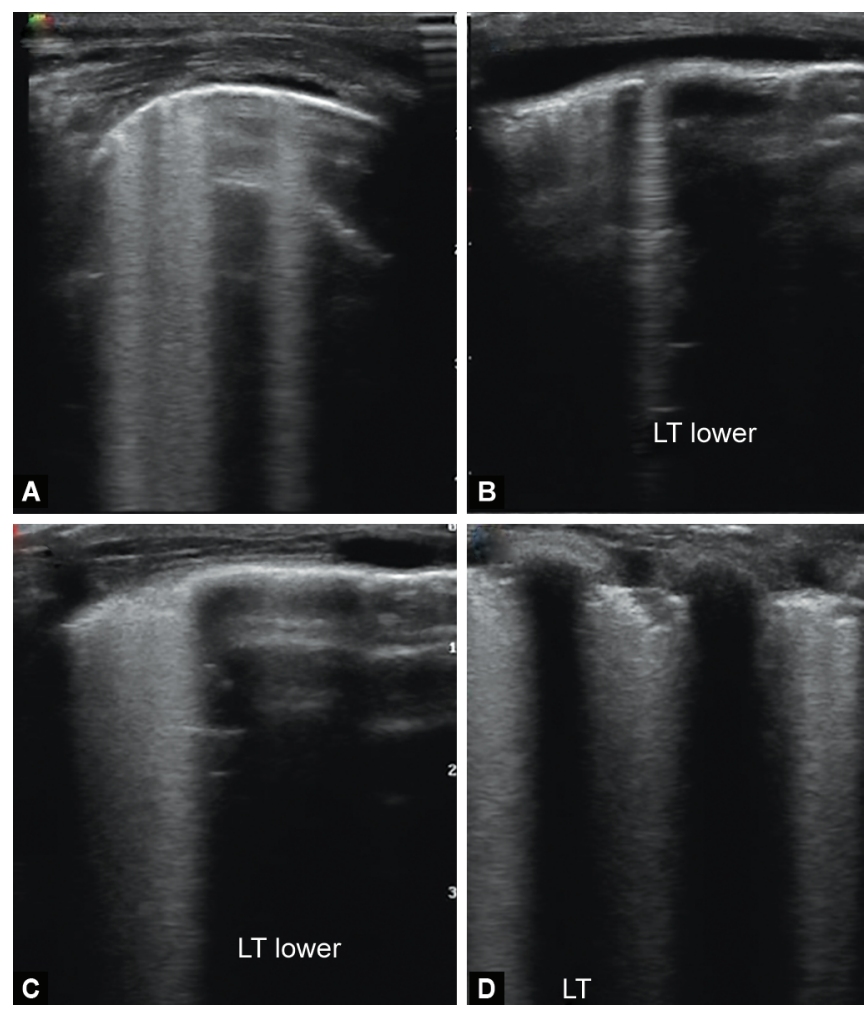

Figs $3 \mathrm{~A}$ to $\mathrm{D}$ : Confluent B-lines

Table 4: Chest X-ray in the studied patients

\begin{tabular}{lcl}
\hline Chest X-ray & No. & (\%) \\
\hline Pneumonia & 28 & 44.4 \\
Pulmonary edema & 11 & 17.5 \\
Not diagnosed (free at examination) & 11 & 17.5 \\
Obstructive lung disease & 7 & 11 \\
Asthma or bronchiolitis & & \\
Pneumothorax & 6 & 9.5 \\
\hline
\end{tabular}

Table 5: Clinical diagnosis in the studied patients

\begin{tabular}{lcc}
\hline Clinical diagnosis & No. & (\%) \\
\hline Pneumonia & 31 & 49.2 \\
Pulmonary edema & 13 & 20.6 \\
Pneumothorax & 8 & 12.7 \\
Asthma & 7 & 11.1 \\
Bronchiolitis & 4 & 6.3 \\
\hline
\end{tabular}

Pereda et al. performed a meta-analysis to confirm evidence on the diagnostic accuracy of LUS for childhood pneumonia. The meta-analysis comprised 765 children. The authors concluded that LUS had a sensitivity of $96 \%$ and specificity of $93 \%$ in accurately diagnosing pneumonia in children as measured against the final diagnosis, while CXR showed a sensitivity of $82 \%$ and a specificity of $94 \%$ in the diagnosis of pneumonia in hospitalized acute respiratory children. ${ }^{9}$ Lichtenstein demonstrated the BLUE protocol performed on the dyspneic patients who will be admitted to the ICU to know the cause of dyspnea in adult critical patients. He showed that the sensitivity of LUS in the diagnosis of pneumonia (four profiles: $C$ profile, B' profile, A with PLAPS, and A/B profile) was $89 \%$, specificity was $94 \%$, PPV was $88 \%$, and NPV was $95 \% .{ }^{6}$ While Lichtenstein and

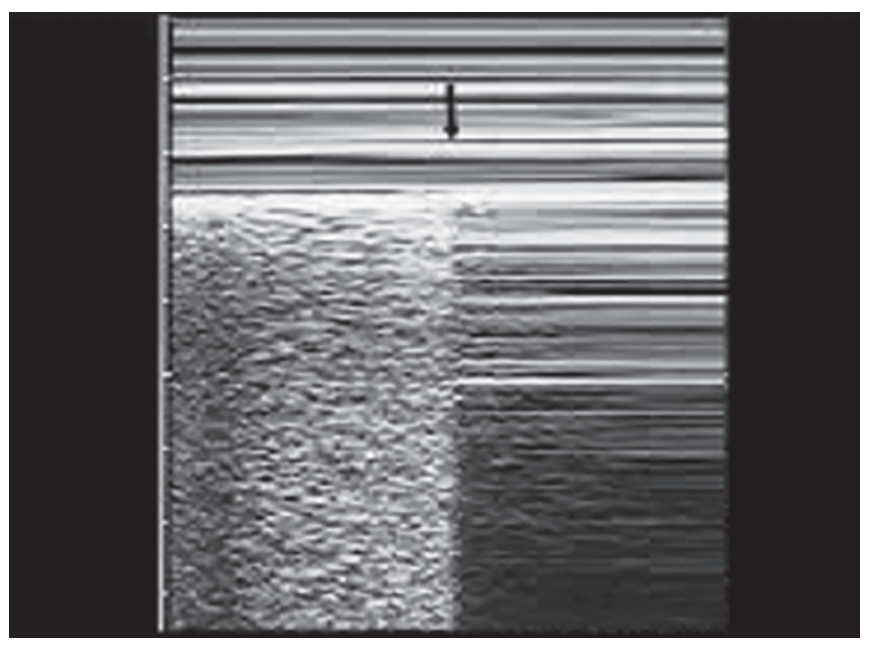

Fig. 4: Lung ultrasound $M$ mode_V2

Mauriat used LUS in critically ill neonate using the BLUE protocol for immediate identification of the cause of acute respiratory failure, they considered that the radiological signs for the main acute disorders are the same in adults and neonates as no radiological distinction has ever been made and demonstrated that LUS sensitivity for alveolar consolidation was $90 \%$ with specificity $98 \%$, while CXR sensitivity was $68 \%$ with specificity $95 \% .{ }^{5}$ Lichtenstein reported that LUS was superior to CXR in the detection of pleural effusion. ${ }^{10}$ Also, another work mentioned that LUS sensitivity, specificity, and diagnostic accuracywere $100 \%$ in the diagnosis of pleural effusion among critically ill patients in comparison with bedside CXR which performed very poorly (sensitivity $65 \%$, specificity $81 \%$, and diagnostic accuracy $69 \% .{ }^{5}$ Xirouchaki et al. and Neto et al. evaluated emergency room patients complaining of dyspnea using LUS; they found it more accurate than CXR in those who subsequently underwent chest CT scans. In Xirouchaki study, the sensitivity and specificity were $100 \%$ compared to a sensitivity of $65 \%$ and specificity $81 \%$ for CXR, while in Neto study, the sensitivity and specificity of LUS for identifying pleural effusion were $90 \%$ and $73 \%$, respectively. ${ }^{11-13}$

In our series, 13 children had a clinical diagnosis of pulmonary edema or interstitial syndrome using BLUE protocol. LUS showed findings consistent with pulmonary edema (B profile) in 14 children as measured against the final diagnosis with a sensitivity of $92.3 \%$, specificity $96 \%$, PPV $85.7 \%$, and NPV 98\%, while CXR was positive for pulmonary edema in 11 children with a sensitivity of $84 \%$, specificity 100\%, PPV 100\%, and NPV 96\%.

Lichtenstein reported that the sensitivity of LUS in detecting the interstitial syndrome (main causes are hemodynamic pulmonary edema and interstitial pneumonia) in critically ill patients was $93 \%$ with a specificity of $93 \%$. He also mentioned that the specificity of LUS using BLUE protocol for the diagnosis of pulmonary edema ( $B$ profile) was $95 \%$. $(6,10,14)$ The same results were confirmed later by Lichtenstein and Mauriat when they used LUS in critically ill neonates but in comparison with CXR that had sensitivity $60 \%$ and specificity $100 \%$ for the interstitial syndrome. ${ }^{5}$ The possible defect of bedside radiography accounts for its principle three dimensions are reduced to two. Alveolar, pleural, and interstitial signs can be challenging to be distinguished, this is overcome by using ultrasound which has three dimensions ability. Neto et al. reported in a study conducted on adults that the diagnostic accuracy of LUS alone was significantly higher than that of CXR alone ( $84 \%$ vs $43 \%){ }^{13}$ 
Efficacy of LUS and CXR in the Diagnosis of Children with Respiratory Distress

Table 6: Detailed performances of the bedside lung ultrasound in emergency protocol in studied children with respiratory distress

\begin{tabular}{|c|c|c|c|c|c|}
\hline Disease & Ultrasound sign used & Sensitivity (\%) & Specificity (\%) & $\begin{array}{l}\text { Positive predictive } \\
\text { value (\%) }\end{array}$ & $\begin{array}{l}\text { Negative predictive } \\
\text { value (\%) }\end{array}$ \\
\hline \multirow[t]{4}{*}{ Pneumonia } & (B' profile) & 93.5 & 96.9 & 96.7 & 93.9 \\
\hline & (A/B profile) & & & & \\
\hline & (C profile) & & & & \\
\hline & A profile plus PLAPS & & & & \\
\hline Pulmonary edema & (B profile) & 92.3 & 96 & 85.7 & 98 \\
\hline Asthma or bronchiolitis & A profile & 90.9 & 98 & 90.9 & 98 \\
\hline Pneumothorax & $\mathrm{A}^{\prime}$ profile with lung point & 100.0 & 100.0 & 100.0 & 100.0 \\
\hline
\end{tabular}

Table 7: Accuracy of chest X-ray

\begin{tabular}{llccl}
\hline & $\begin{array}{l}\text { Sensitivity } \\
\text { (\%) }\end{array}$ & $\begin{array}{l}\text { Specificity } \\
\text { (\%) }\end{array}$ & $\begin{array}{l}\text { Positive } \\
\text { predictive } \\
\text { value (\%) }\end{array}$ & $\begin{array}{l}\text { Negative } \\
\text { predictive } \\
\text { value (\%) }\end{array}$ \\
\hline Disease & 90.3 & 87.7 & 87.5 & 90.3 \\
Pneumonia & 84.6 & 100.0 & 100.0 & 96.1 \\
$\begin{array}{l}\text { Obstmonary edema } \\
\text { disease (asthma or } \\
\text { bronchiolitis) }\end{array}$ & 54.5 & 98.0 & 85.7 & 91.0 \\
Pneumothorax & 75.0 & 100.0 & 100.0 & 96.7 \\
\hline
\end{tabular}

Silva et al. demonstrated that the diagnostic accuracy of the LUS approach in acute respiratory failure patients was higher than that of an initial routine evaluation based on clinical, radiological, and biological data $(83 \%$ vs $63 \%, p<0.02)$. The most common etiologies for RD were pneumonia and hemodynamic lung edema. They concluded that LUS based on the BLUE protocol was reproducible by physicians who are not LUS experts and accurate for the diagnosis of pneumonia and hemodynamic pulmonary edema. ${ }^{15}$

In our study, using BLUE protocol, LUS showed findings consistent with asthma or bronchiolitis in 11 children (A with no $B$ nor PLAPS profile) with a sensitivity of $90.9 \%$, specificity $98 \%$, PPV $90.9 \%$, and NPV $98 \%$ as measured against final diagnosis, while CXR was positive for asthma or bronchiolitis only in 7 children with a sensitivity of $54.5 \%$, specificity $98 \%$, PPV $85.7 \%$, and NPV $91 \%$. Two previous studies, conducted on critically ill adult patients, published that the sensitivity of LUS using BLUE protocol was $89 \%$ with a specificity of $97 \%$, PPV 93\%, and NPV 95\% in the diagnosis of asthma or chronic obstructive pulmonary disease (COPD). ${ }^{6,14}$

According to Basile et al., the agreement between the clinical and sonographic diagnoses in infants with bronchiolitis was good $90.6 \%$ with a statistically significant interobserver ultrasound diagnosis concordance of $89.6 \%$. The LUS correlated to clinical evaluation and permitted the identification of infants in need of supplemental oxygen with high specificity. ${ }^{16}$

In the present study, of the eight children with a confirmed diagnosis of pneumothorax using BLUE protocol, LUS showed findings consistent with pneumothorax in all of them $\left(A^{\prime}\right.$ profile with lung point) with a sensitivity of $100 \%$, specificity $100 \%$, PPV $100.0 \%$, and NPV $100 \%$, while CXR was positive for pneumothorax in six children with a sensitivity of $75 \%$, specificity $100 \%$, PPV $100 \%$, and NPV 96.4\%.

Raimondi et al. found that the accuracy of LUS in diagnosing pneumothorax was quite high reaching $100 \%$ in critically ill neonates. ${ }^{17}$ Lichtenstein found that the sensitivity of LUS using BLUE protocol in the diagnosis of pneumothorax was $88 \%$ with a specificity of $100 \%$, PPV $100 \%$, and NPV $99 \%$. In critically ill adult patients, the detection of lung points confirms pneumothorax diagnosis. 6,14

Given this low sample size in one center, we were not able to exclude type II error, and this requires further research to check the validity of results. Also, findings and their interpretations are operator dependent and choice of the probe is critical so ultrasound interpretation should always occur in the context of the clinical condition.

\section{Conclusion}

The LUS using BLUE protocol allows fast and accurate bedside diagnosis of acute respiratory illnesses in children in comparison with CXR. It enables a pathophysiological approach to identify etiology. The versatility of LUS in visual medicine gives it a priority in intensive care emergency settings as well as many other disciplines and settings.

\section{Ethical Approval}

All procedures performed in the studies involving human participants were under the ethical standards of Ain Shams University Faculty of Medicine Research Ethics Board (Reference number 21737) and with the 1964 Declaration of Helsinki and its later amendments or comparable ethical standards.

This article does not contain any studies with animals performed by any of the authors.

\section{References}

1. Warren J, Fromm REJ, Orr RA, Rotello LC, Horst HM. Guidelines for the inter- and intrahospital transport of critically ill patients. Crit Care Med 2004;32(1):256-262. DOI: 10.1097/01.CCM.0000104917. 39204.0A.

2. Baldi G, Gargani L, Abramo A, D'Errico L, Caramella D, Picano E, et al. Lung water assessment by lung ultrasonography in intensive care: a pilot study. Intensive Care Med 2013;39(1):74-84. DOI: 10.1007/ s00134-012-2694-x.

3. Volpicelli G, El Barbary M, Blaivas M, Lichtenstein D, Mathis G, Kirkpatrick AW, et al. International evidence-based recommendations for point-of-care lung ultrasound. Intensive Care Med [Internet] 2012. 38. DOI: 10.1007/s00134-012-2513-4.

4. Lichtenstein DA, Meziere GA. Relevance of lung ultrasound in the diagnosis of acute respiratory failure: the BLUE protocol. Chest 2008;134(1):117-125. DOI: 10.1378/chest.07-2800.

5. Lichtenstein $D$, Mauriat P. Lung ultrasound in the critically ill neonate. Curr Pediatr Rev [Internet] 2012;8(3).DOI: 10.2174/157339612802139389.

6. Lichtenstein DA. Lung ultrasound in the critically ill. Ann Intensive Care [Internet] 2014;4(1):1. DOI: 10.1186/2110-5820-4-1.

7. Krishnan S, Moghekar A, Duggal A, Yella J, Narechania S, Ramachandran V, et al. Radiation exposure in the medical ICU: 
predictors and characteristics. Chest 2018;153(5):1160-1168. DOI: 10.1016/j.chest.2018.01.019.

8. Reali F, Sferrazza Papa GF, Carlucci P, Fracasso P, Di Marco F, Mandelli $M$, et al. Can lung ultrasound replace chest radiography for the diagnosis of pneumonia in hospitalized children? Respiration 2014;88(2):112-115. DOI: 10.1159/000362692.

9. Pereda MA, Chavez MA, Hooper-Miele CC, Gilman RH, Steinhoff MC, Ellington $\mathrm{LE}$, et al. Lung ultrasound for the diagnosis of pneumonia in children: a meta-analysis. Pediatrics 2015;135(4):714-722. DOI: 10.1542/peds.2014-2833.

10. Lichtenstein DA. Ultrasound examination of the lungs in the intensive care unit. Pediatr Crit Care Med 2009;10(6):693-698. DOI: 10.1097/ PCC.0b013e3181b7f637.

11. Xirouchaki N, Magkanas E, Vaporidi K, Kondili E, Plataki M, Patrianakos $A$, et al. Lung ultrasound in critically ill patients: comparison with bedside chest radiography. Intensive Care Med 2011;37(9):1488-1493. DOI: 10.1007/s00134-011-2317-y.

12. Dexheimer Neto FL, Andrade JMS, Raupp ACT, Townsend RS, Beltrami FG, Brisson $\mathrm{H}$, et al. Diagnostic accuracy of the bedside lung ultrasound in emergency protocol for the diagnosis of acute respiratory failure in spontaneously breathing patients. J Bras
Pneumol Publicacao of da Soc Bras Pneumol e Tisilogia 2015;41(1) 58-64. DOI: 10.1590/S1806-37132015000100008.

13. Dexheimer Neto FL, Dalcin P, de TR, Teixeira C, Beltrami FG. Lung ultrasound in critically ill patients: a new diagnostic tool. J Bras Pneumol Publicacao of da Soc Bras Pneumol e Tisilogia 2012;38(2):246-256. DOI: 10.1590/s1806-37132012000200015.

14. Lichtenstein DA. BLUE-protocol and FALLS-protocol: two applications of lung ultrasound in the critically ill. Chest 2015;147(6):1659-1670. DOI: 10.1378/chest.14-1313.

15. Silva S, Biendel C, Ruiz J, Olivier M, Bataille B, Geeraerts T, et al. Usefulness of cardiothoracic chest ultrasound in the management of acute respiratory failure in critical care practice. Chest 2013;144(3):859-865. DOI: 10.1378/chest.13-0167.

16. Basile V, Di Mauro A, Scalini E, Comes P, Lofù I, Mostert M, et al. Lung ultrasound: a useful tool in diagnosis and management of bronchiolitis. BMC Pediatr [Internet] 2015;15(1):63. DOI: 10.1186/s12887-015-03801Available from: https://www.ncbi.nlm.nih.gov/pubmed/25993984.

17. Raimondi F, Rodriguez Fanjul J, Aversa S, Chirico G, Yousef N, De Luca $D$, et al. Lung ultrasound for diagnosing pneumothorax in the critically III neonate. J Pediatr 2016;175:74-78.e1. DOI: 10.1016/ j.jpeds.2016.04.018. 